\title{
Metformin use in patients with type 2 diabetes mellitus and chronic kidney disease: An evidence-based review
}

Felicia Clara JH Tan, ${ }^{1} M B$ BChir (Cantab), Seng Bin Ang, ${ }^{1,2}{ }_{F C F P(\text { Singapore), Yong Mong Bee }}{ }^{2,3}{ }_{F R C P(E d i n)}$

\begin{abstract}
Introduction: Practice guidelines advise caution on the use of metformin in patients with type 2 diabetes mellitus with chronic kidney disease (CKD). This review aims to examine the evidence for the benefits and risks of metformin use in patients with T2DM and CKD.

Methods: The Cochrane Database of Systematic Reviews, the Cochrane Central Register of Controlled Trials and PubMed were searched; the references of selected papers were hand searched. Systematic reviews, randomised controlled trials, cohort studies, case series and case-control studies were included. The full text of selected articles was reviewed. The outcomes studied were all-cause mortality, cardiovascular complications, lactic acidosis and worsening of renal function. Recommendations were graded according to the Scottish Intercollegiate Guidelines Network system.

Results: A total of 139 unique articles were identified, 14 of which met the inclusion criteria and were selected for full-text review. Four cohort studies reported an association between metformin use and improved all-cause mortality in CKD stage 4 and better. Two cohort studies reported improved cardiovascular outcomes with metformin use. Four cohort studies, 1 case series and 1 case-control study reported no significant association between metformin use and an increased risk of lactic acidosis in CKD. There is a moderate level of evidence to support reduced mortality, improved cardiovascular outcomes and a low risk of lactic acidosis with metformin use in patients with T2DM and with CKD stage 4 and above.

Conclusion: Existing recommendations to restrict metformin use in diabetes patients with CKD need to be reviewed in light of emerging evidence supporting its overall benefits in these patients.
\end{abstract}

Ann Acad Med Singap 2021;50:159-70

Keywords: Chronic renal insufficiency, metformin, type 2 diabetes mellitus

\section{INTRODUCTION}

Diabetes is a chronic disease characterised by elevated levels of blood glucose. The most common type of diabetes is type 2 diabetes mellitus (T2DM). This usually occurs in adults and arises through insulin resistance or an insufficiency of insulin production. ${ }^{1}$ Diabetes is one of the priority non-communicable diseases targeted for action by world leaders. Its prevalence has been steadily increasing over the last few decades; the global prevalence of diabetes in the adult population has risen from $4.7 \%$ in 1980 to $8.5 \%$ in 2014. ${ }^{2}$ Chronic kidney disease (CKD) describes abnormal kidney function, abnormal kidney structure, or both. The severity of CKD can be determined by glomerular filtration rate and the presence of markers of kidney damage, such as albuminuria, urine sediment abnormalities, electrolyte abnormalities, abnormalities caused by tubular disease, structural abnormalities and abnormalities detected by histology. ${ }^{3}$ The stages of CKD are shown in Table 1.

CKD can arise as a consequence of poorly controlled diabetes. The increasing incidence of diabetic kidney disease is the key driver of the burden of CKD worldwide. The prevalence of diabetic kidney disease has increased by $39.5 \%$ globally between 2005 and 2015. In Mexico, the country with the highest death rate from CKD in the world, more than half of all cases of end-stage kidney disease were attributed to diabetes. ${ }^{4}$ Slowing the progression of diabetic kidney disease requires glycaemic control. This can be achieved with

\footnotetext{
${ }^{1}$ Family Medicine Service, KK Women's and Children's Hospital, Singapore

${ }^{2}$ Duke-NUS Medical School, Singapore

${ }^{3}$ Department of Endocrinology, Singapore General Hospital, Singapore

Correspondence: Dr Felicia Clara Tan, Family Medicine Service, KK Women's and Children's Hospital, 100 Bukit Timah Road, Singapore 229899.

E-mail: feliciaclara.tan@mohh.com.sg
} 


\section{CLINICAL IMPACT}

\section{What is New}

- Metformin use is associated with reduced mortality and improved cardiovascular outcomes in chronic kidney disease (CKD) even as severe as stage 4 while the risk of lactic acidosis is low.

\section{Clinical Implications}

- Metformin, the first-line oral hypoglycaemic agent for type 2 diabetes in almost all guidelines worldwide, has its use restricted in stage 3-5 CKD for fear of lactic acidosis. This review calls for reconsideration in restricting metformin use in type 2 diabetic patients with CKD stage 3 and 4 .

Table 1. Stages of chronic kidney disease

\begin{tabular}{|c|c|}
\hline Stage & Glomerular filtration rate \\
\hline 1 & $\geq 90$ with other markers of kidney damage \\
\hline 2 & $60-89$ with other markers of kidney damage \\
\hline $3 a$ & $45-59$ \\
\hline $3 b$ & $30-44$ \\
\hline 4 & $15-29$ \\
\hline 5 & $<15$ \\
\hline \multicolumn{2}{|c|}{$\begin{array}{l}\text { 3a and 3b Source: National Institute for Health and Care Excellence. } \\
\text { Chronic kidney disease in adults: assessment and management. } \\
\text { Clinical guideline, } 23 \text { July 2014. Available at: https://www.nice.org.uk/ } \\
\text { guidance/cg182. Accessed on } 24 \text { April } 2020 \text {. }\end{array}$} \\
\hline
\end{tabular}

lifestyle changes and a variety of medications, including oral glucose-lowering medications and insulin. Metformin is the current first-line pharmacological treatment for type 2 diabetes in almost all recommendations worldwide. It is an orally administered drug belonging to the biguanide class of glucose-lowering medication. ${ }^{5}$ It decreases liver glucose production and intestinal absorption of glucose and increases insulin sensitivity, thereby decreasing blood glucose levels. It reduces both basal and postprandial blood glucose. ${ }^{6}$ Metformin is primarily renally eliminated; owing to genetic polymorphisms (e.g. in the organic cation transporters mediating metformin transmembrane transport), there is considerable variation in the renal clearance of metformin. ${ }^{7}$

Existing guidelines recommend using a reduced dose of metformin or ceasing it in renal impairment because of a lack of evidence for the safety of metformin in renal impairment and the concern of lactic acidosis. The National Institute for Health and Care Excellence (NICE) guidelines recommend that the dose of metformin be reviewed when the estimated glomerular filtration rate (eGFR) drops below $45 \mathrm{~mL} / \mathrm{min} / 1.73 \mathrm{~m}^{2}$, and metformin be stopped altogether if the eGFR falls below $30 \mathrm{~mL} / \mathrm{min} / 1.73 \mathrm{~m}^{2}$. These recommendations were made in view of a lack of evidence for the safety of metformin in people with eGFR less than $30 \mathrm{~mL} /$ $\min / 1.73 \mathrm{~m}^{2}{ }^{8}$

Similar to the NICE guidelines, Singapore's Ministry of Health clinical practice guidelines for T2DM advise that metformin use be reviewed in those with eGFR less than $45 \mathrm{~mL} / \mathrm{min} / 1.73 \mathrm{~m}^{2}$ (stage $3 \mathrm{~b}$ ) and ceased if the eGFR is less than $30 \mathrm{~mL} / \mathrm{min} / 1.73 \mathrm{~m}^{2}$ (stage 4). Metformin is usually contraindicated in severe renal impairment as it may be associated with lactic acidosis. ${ }^{9}$ However, the evidence for these recommendations is not strong; it was given a grade D, level 4 rating. The American Association of Clinical Endocrinologists and the American College of Endocrinology also recommend a reduction in metformin dose for patients whose eGFR is between $30 \mathrm{~mL} / \mathrm{min} / 1.73 \mathrm{~m}^{2}$ and $45 \mathrm{~mL} / \mathrm{min} / 1.73 \mathrm{~m}^{2}$ (stage $3 \mathrm{~b}$ ), and a cessation of metformin in those whose eGFR is below $30 \mathrm{~mL} / \mathrm{min} / 1.73 \mathrm{~m}^{2}$ (stage 4 ). ${ }^{10}$

These recommendations for reducing the dose of or ceasing metformin were based on the lack of evidence for its safety in a population with renal impairment. There have been suggestions that the risks of metformin use in renally impaired patients are overstated. By restricting the use of metformin in populations with renal impairment, they might be deprived of the benefits of metformin, ${ }^{11}$ and be exposed unnecessarily to the risks of using other glucose-lowering medications. For example, if their dose of metformin is reduced or stopped, they may have to increase their dose of sulfonylureas, which puts them at greater risk of hypoglycaemic events, ${ }^{12}$ or increase their dose of insulin, which may increase their weight gain. ${ }^{13}$ Metformin use has been suggested to be associated with a host of clinical benefits, including a reduction in mortality,${ }^{14}$ a reduction in cardiovascular complications ${ }^{15}$ and benefits for vascular function. ${ }^{14}$ Additionally, metformin has shown potential renoprotective effects against diabetic nephropathy in both in vitro and animal models. Under high glucose conditions, metformin modulates apoptosis and cell signalling of human podocytes. It therefore reduces the loss of podocytes, which is a key process in diabetic nephropathy. In 
animal studies, diabetic rats that were treated with metformin had a significant dose-dependent reduction in urinary albumin and nephrin concentration, glomerular basement membrane thickness and the rate of foot process fusion compared with diabetic rats not given metformin. ${ }^{16}$ This review aims to evaluate the quality of the available evidence regarding the benefits of metformin (such as improvement in mortality, reduction in cardiovascular events) and their adverse effects (such as incidence of lactic acidosis, worsening of renal function) in adults with T2DM and CKD.

\section{METHODS}

Searches were made of the Cochrane Database of Systematic Reviews (CDSR), the Cochrane Central Register of Controlled Trials (CCRCT) and PubMed on 5 November 2020. The search query employed for CDSR and CCRCT was ("lactic acidosis":ti,ab,kw OR "cardiovascular events":ti,ab,kw OR "mortality":ti,ab,kw) AND (metformin:ti,ab,kw) AND ("chronic kidney disease":ti,ab,kw OR "chronic renal insufficiency":ti,ab,kw) AND (diabetes:ti,ab,kw). For PubMed, the search query was ("lactic acidosis" [tiab] OR "cardiovascular events" [tiab] OR mortality [tiab]) AND (metformin [tiab]) AND ("chronic kidney disease" [tiab] OR "chronic renal insufficiency" [tiab]) AND (diabetes [tiab]).

The databases were searched from inception to 5 November 2020. Search results were limited to articles available in English. In addition, the reference lists of selected articles identified from database searching were hand searched for suitable articles.

To be included in this review, the articles had to meet the following criteria:

- Type of article: a systematic review, randomised controlled trial, cohort study, case-control study or case series.

- Population studied in the article: adult humans with T2DM and CKD taking metformin. Only articles that studied CKD as their main focus were included.

- Outcome studied in the article must be one or more of the following: improvements in mortality, rate of cardiovascular events, incidence of lactic acidosis, or worsening of renal function.

- Full text in English that was readily available.

Database searching yielded 102 records from PubMed and 0 records from CDSR and CCRCT. Hand searching of the reference lists of selected articles identified from database searching yielded 66 records. After duplicates were removed, 139 unique articles were identified. Their titles and abstracts were screened for inclusion. A total of 14 articles met the inclusion criteria above and were included for final analysis. The process of article selection is shown in Fig. 1. A summary of the articles and their findings is presented in Table 2 .

Each article, including systematic reviews, was graded for quality using the Scottish Intercollegiate Guidelines Network (SIGN) tools for critical appraisal (available at https://www.sign.ac.uk/what-we-do/ methodology/checklists/). The quality ratings were considered in order to form an overall grade for the strength of evidence for each outcome. The grading system from SIGN that was used to grade the strength of evidence is presented in Table 3. All articles except the systematic reviews were then included for narrative synthesis. The systematic reviews were excluded from narrative synthesis to avoid certain articles that had been included in this review and in the selected systematic reviews from being included twice.

\section{RESULTS}

The grading of evidence for each of the outcomes is summarised in Table 4. A summary of outcomes and CKD stage investigated in each article is provided in Table 5.

\section{Quality of evidence}

Of the 14 articles included for analysis, 3 were systematic reviews, ${ }^{17-19} 9$ were cohort studies ${ }^{20-28}$ (8 of which were retrospective cohort studies ${ }^{20-27}$ and 1 was a post hoc analysis of a $\operatorname{trial}^{28}$ ), 1 was a case-control study $^{29}$ and 1 was a case series. ${ }^{30}$ Three studies ${ }^{27,28,30}$ involved participants in specialist centres, while 3 studies $^{24-26}$ involved participants from primary care. Six studies analysed data from databases without making distinctions between patients who were seen in primary care and those who were seen in specialist care. ${ }^{19-23,29}$

The quality of the review by Crowley et al. ${ }^{17}$ was rated as "minus" (-) because of the lack of a table of baseline characteristics of participants. The reviews by $\mathrm{Lu}$ et al. ${ }^{18}$ and $\mathrm{Hu}$ et al. ${ }^{19}$ demonstrated an overall acceptable quality and were rated as "plus" $(+)$. One review by Inzucchi et al. ${ }^{31}$ was excluded from analysis after full-text review as its quality was deemed unacceptable. It suffered from serious flaws including the lack of 2 reviewers performing the literature search and data extraction, and the lack of quality assessment of included studies.

The quality of the cohort studies were acceptable with the exception of the cohort studies by Ekström et al., ${ }^{23} \mathrm{Hsu}$ et al. ${ }^{27}$ and Richy et al. ${ }^{26}$ that were marked 


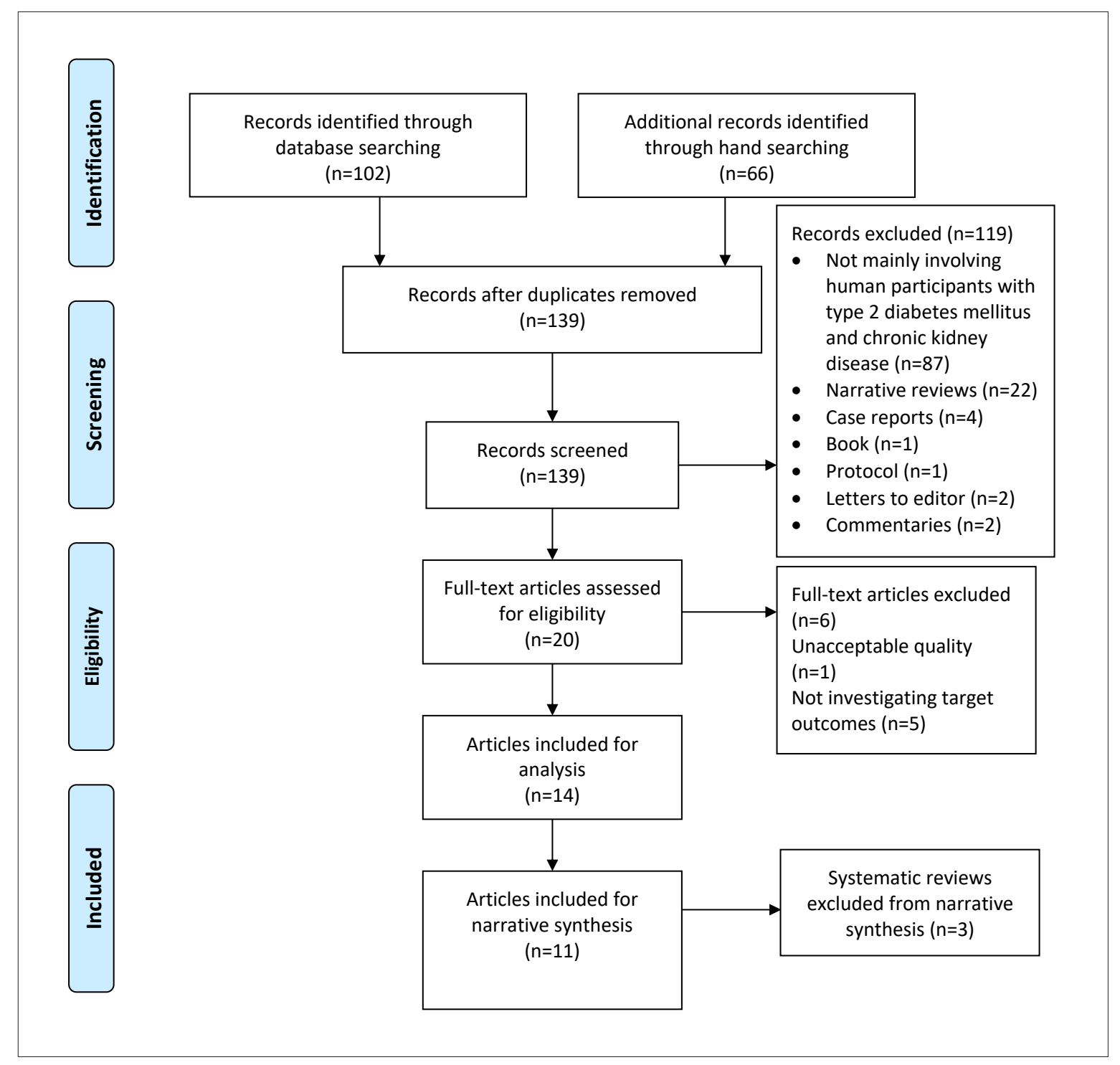

Fig. 1. Flow diagram showing process of article selection.

down for a lack of sensitivity analysis and failure to account for confounding. Common flaws among the cohort studies were the lack of blinding and lack of sensitivity analysis.

The level of evidence for some cohort studies was also limited by virtue of them being retrospective studies. Retrospective cohort studies may be subject to various biases, including information bias ${ }^{32}$ and selection bias. ${ }^{33}$ Additionally, cohort studies may also suffer from confounding by indication. ${ }^{34}$

\section{All-cause mortality}

Four cohort studies ${ }^{20,21,23,28}$ found an association between metformin use and improved all-cause mortality in adults with T2DM and CKD. Whitlock et al. ${ }^{20}$ reported a lower risk of mortality for T2DM patients with stage 2 renal impairment using metformin than for those using sulfonylureas, while there was no significant improvement in mortality found for T2DM patients with CKD stage $3 \mathrm{a}$ and below. Charytan et al. ${ }^{28}$ reported reduced mortality for patients with CKD stages 2-4 using metformin compared with non-users. Marcum et al. ${ }^{21}$ reported reduced mortality for those with CKD stages 1-3a using metformin compared with those using sulfonylureas. Ekström et al. ${ }^{23}$ found a reduced all-risk mortality in patients with CKD stage 3a using metformin compared with those using other oral glucose-lowering medications. In contrast, Hung et al. ${ }^{22}$ reported increased all-cause mortality in T2DM patients with CKD stage 5 . 


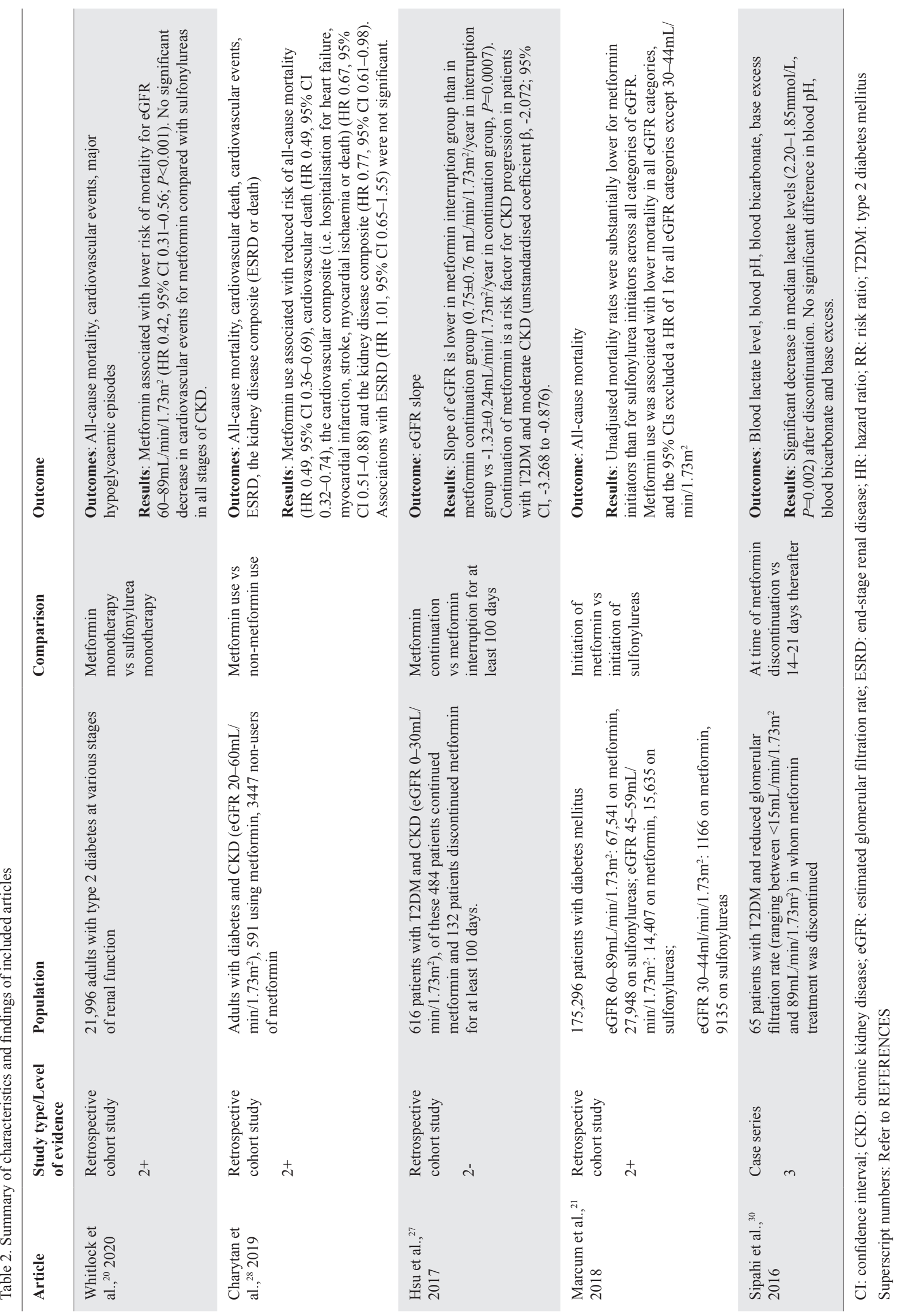




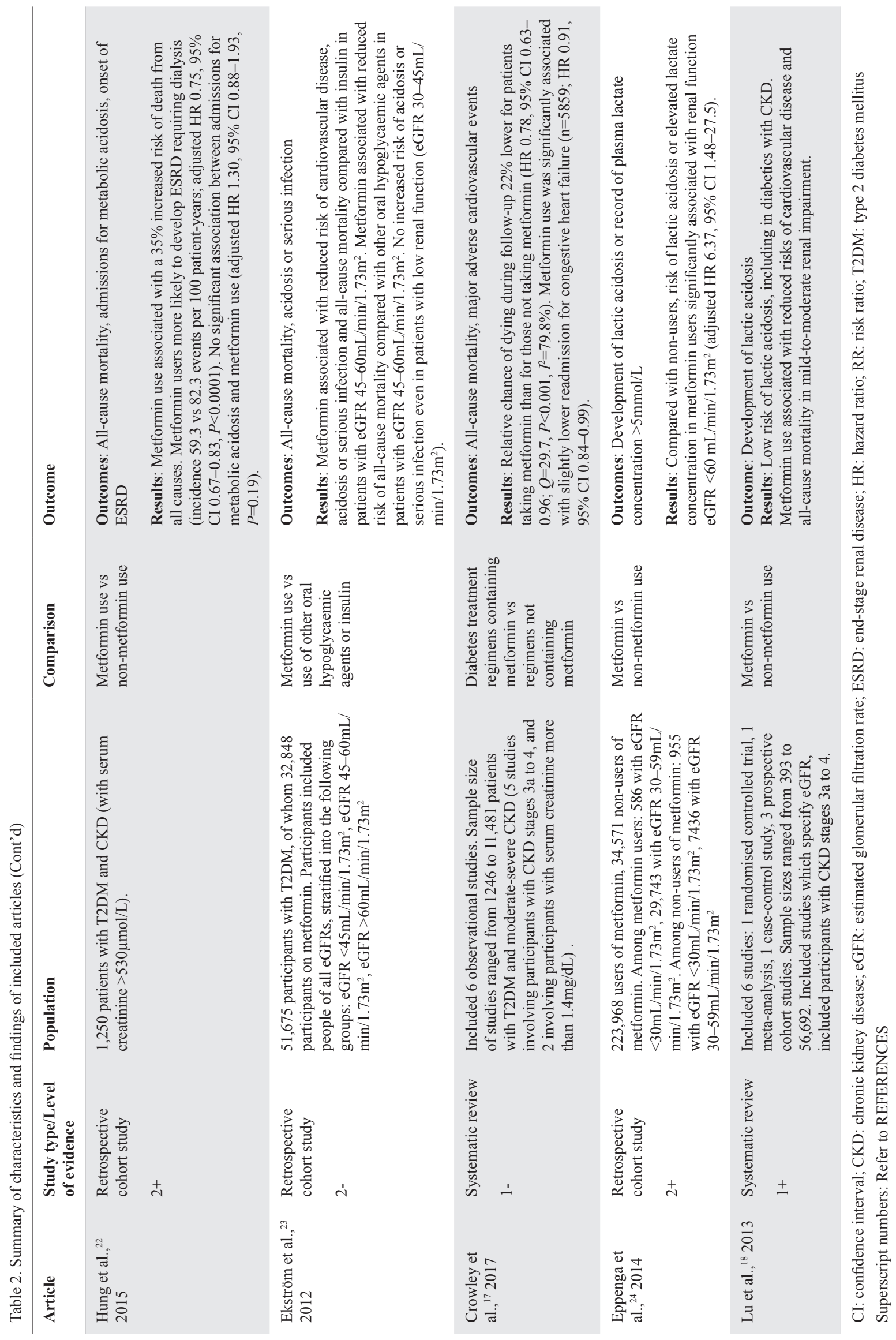




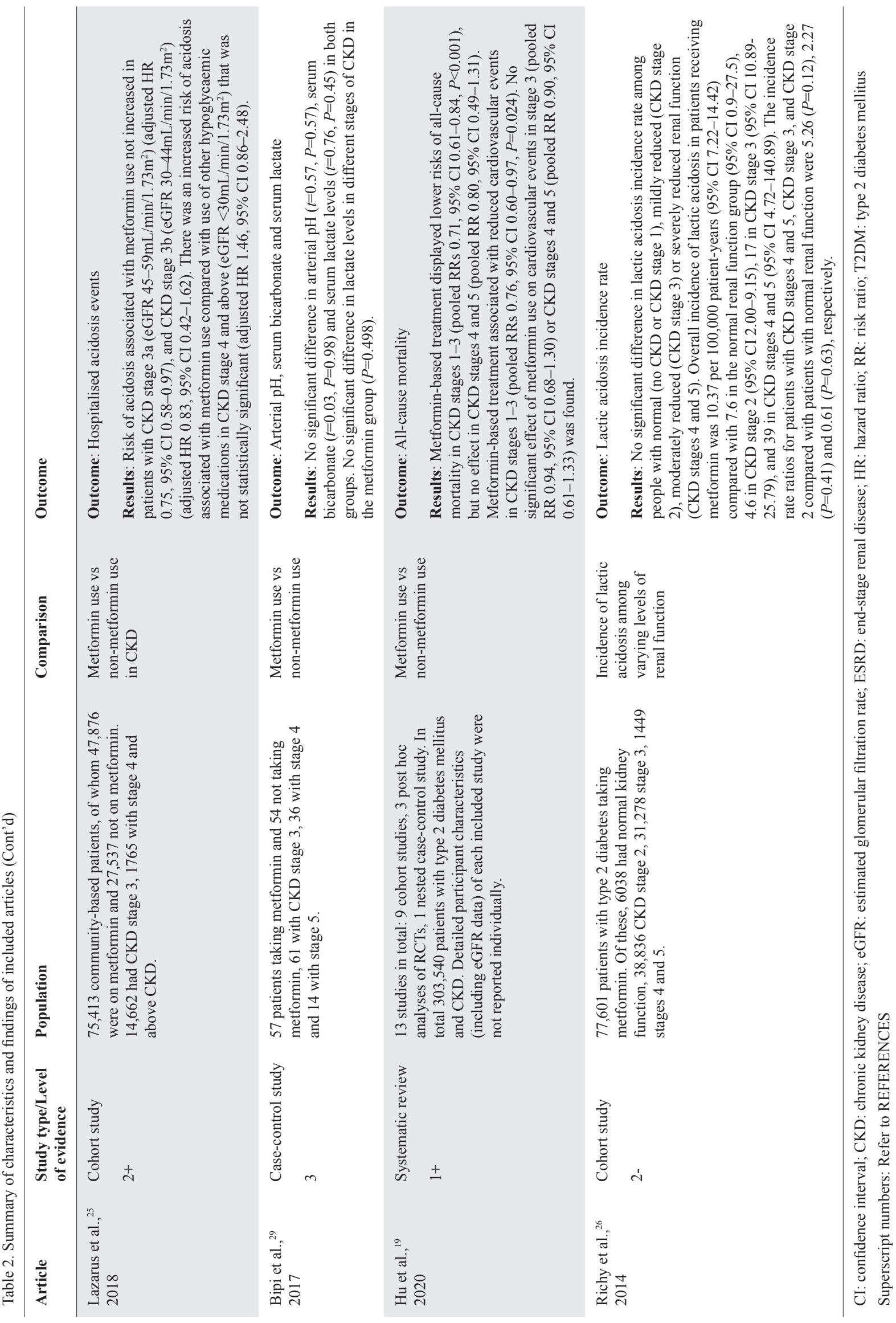


Table 3. Summary of the Scottish Intercollegiate Guidelines Network grading system ${ }^{38}$

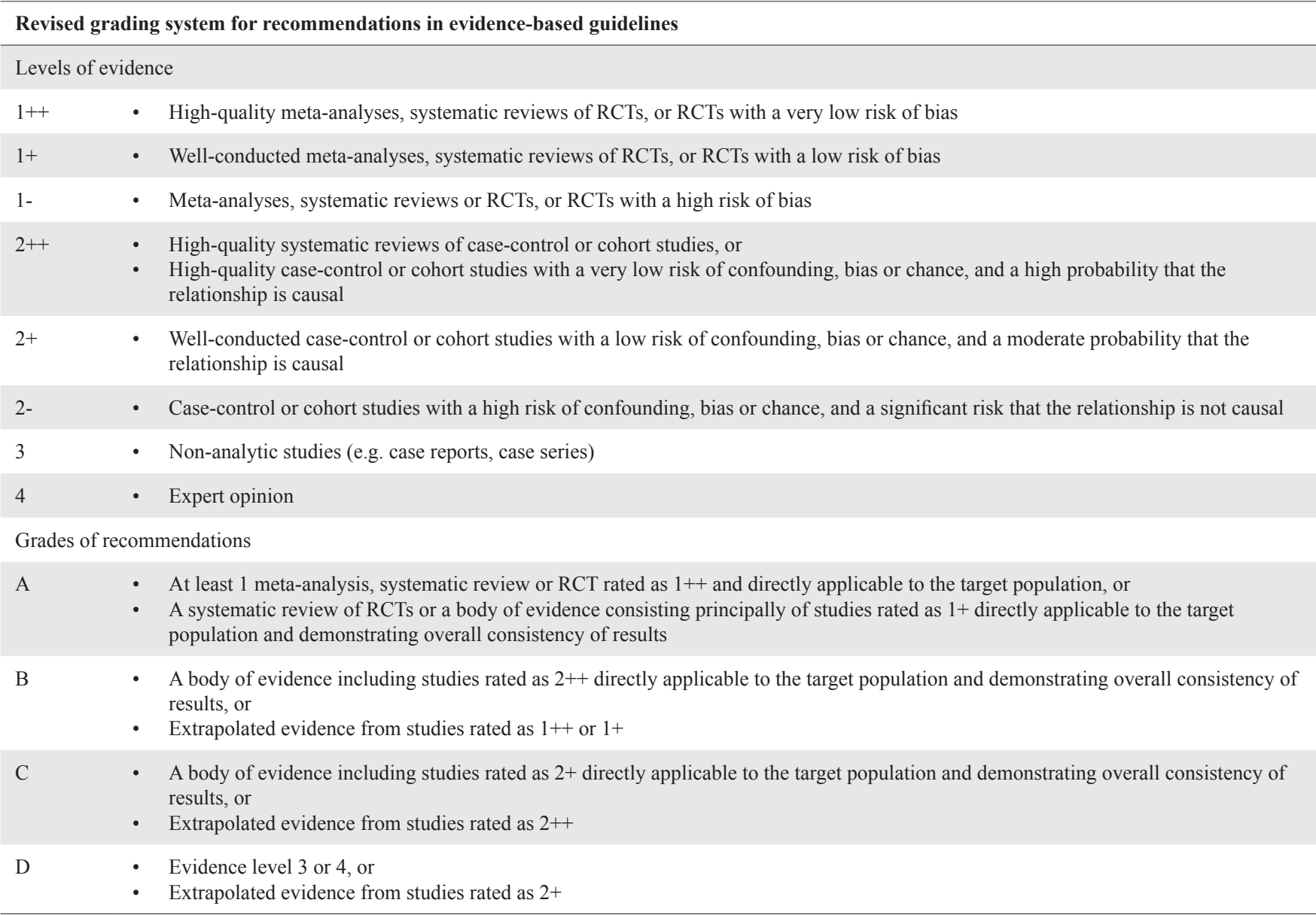

RCT: randomised controlled trial

Table 4. Summary of grading of evidence supporting each outcome in the use of metformin in chronic kidney disease

\begin{tabular}{|c|c|c|}
\hline Recommendation & Evidence grading & References \\
\hline Metformin is associated with reduced all-cause mortality & $\mathrm{B}, 1+$ & $19,20,21,23,28$ \\
\hline Metformin is not associated with increased risk of lactic acidosis & $\mathrm{C}, 1-$ & $22,23,25,26,29,30$ \\
\hline Metformin is not associated with increased risk of worsening renal function & $\mathrm{D}, 2+$ & 28 \\
\hline Metformin is associated with increased risk of worsening renal function & $\mathrm{C}, 2+$ & 22,27 \\
\hline
\end{tabular}

\section{Cardiovascular complications}

Two cohort studies ${ }^{23,28}$ found improved cardiovascular outcomes with the use of metformin. Charytan et al. ${ }^{28}$ reported that metformin use in patients with CKD stages 2-4 was associated with reduced risk of cardiovascular death and cardiovascular complications (i.e. hospitalisation for heart failure, myocardial infarction, stroke, myocardial ischaemia or death). Ekström et al. ${ }^{23}$ similarly reported that metformin use was associated with a reduced risk of cardiovascular diseases in patients with CKD stage $3 \mathrm{a}$. In contrast, Whitlock et al. ${ }^{20}$ found that metformin use was not significantly associated with a decrease in cardiovascular events in patients with CKD compared with sulfonylurea use.

\section{Lactic acidosis}

One case series, ${ }^{30} 5$ cohort studies ${ }^{22-26}$ and 1 case-control study $^{29}$ investigated the association between metformin use and lactic or metabolic acidosis. Sipahi et al. ${ }^{30}$ 
Table 5. Summary of outcomes by estimated glomerular filtration rate (eGFR) from each article

\begin{tabular}{|c|c|}
\hline Outcome & $\begin{array}{l}\text { Articles reporting this outcome (for which eGFR information } \\
\text { is available) }\end{array}$ \\
\hline \multicolumn{2}{|c|}{ Metformin is associated with improved all-cause mortality } \\
\hline eGFR $60-89 \mathrm{~mL} / \mathrm{min} / 1.73 \mathrm{~m}^{2}$ & Whitlock et al., ${ }^{20}$ Charytan et al., ${ }^{28}$ Marcum et al., ${ }^{21}$ Hu et al., ${ }^{19}$ \\
\hline eGFR $45-59 \mathrm{~mL} / \mathrm{min} / 1.73 \mathrm{~m}^{2}$ & Charytan et al., ${ }^{28}$ Marcum et al., ${ }^{21}$ Ekström et al., ${ }^{23}$ Hu et al., ${ }^{19}$ \\
\hline eGFR $30-45 \mathrm{~mL} / \mathrm{min} / 1.73 \mathrm{~m}^{2}$ & Charytan et al., ${ }^{28}$ Marcum et al., ${ }^{21} \mathrm{Hu}$ et al., ${ }^{19}$ \\
\hline eGFR $15-30 \mathrm{~mL} / \mathrm{min} / 1.73 \mathrm{~m}^{2}$ & Charytan et al., ${ }^{28}$ Marcum et al., ${ }^{21}$ \\
\hline \multicolumn{2}{|c|}{ Metformin is associated with improved cardiovascular outcomes } \\
\hline eGFR $60-89 \mathrm{~mL} / \mathrm{min} / 1.73 \mathrm{~m}^{2}$ & Charytan et al., ${ }^{28}$ \\
\hline eGFR $45-59 \mathrm{~mL} / \mathrm{min} / 1.73 \mathrm{~m}^{2}$ & Charytan et al., ${ }^{28}$ \\
\hline eGFR $15-30 \mathrm{~mL} / \mathrm{min} / 1.73 \mathrm{~m}^{2}$ & Charytan et al., ${ }^{28}$ \\
\hline \multicolumn{2}{|c|}{ Metformin is not associated with increased risk of lactic acidosis } \\
\hline eGFR $60-89 \mathrm{~mL} / \mathrm{min} / 1.73 \mathrm{~m}^{2}$ & Lu et al., ${ }^{18}$ Richy et al., ${ }^{26}$ \\
\hline eGFR $45-59 \mathrm{~mL} / \mathrm{min} / 1.73 \mathrm{~m}^{2}$ & Ekström et al., ${ }^{23}$ Lu et al., ${ }^{18}$ Lazarus et al., ${ }^{25}$ Bipi et al., ${ }^{29}$ Richy et al., ${ }^{26}$ \\
\hline eGFR $30-45 \mathrm{~mL} / \mathrm{min} / 1.73 \mathrm{~m}^{2}$ & Ekström et al., ${ }^{23}$ Lazarus et al., ${ }^{25}$ Bipi et al., ${ }^{29}$ Richy et al., ${ }^{26}$ \\
\hline eGFR $15-29 \mathrm{~mL} / \mathrm{min} / 1.73 \mathrm{~m}^{2}$ & Lazarus et al., ${ }^{25}$ Bipi et al., ${ }^{29}$ Richy et al., ${ }^{26}$ \\
\hline $\mathrm{eGFR}<15 \mathrm{~mL} / \mathrm{min} / 1.73 \mathrm{~m}^{2}$ & Lazarus et al., ${ }^{25}$ Bipi et al., ${ }^{29}$ Richy et al., ${ }^{26}$ \\
\hline \multicolumn{2}{|c|}{ Metformin is not associated with increased risk of worsening renal function } \\
\hline eGFR $60-89 \mathrm{~mL} / \mathrm{min} / 1.73 \mathrm{~m}^{2}$ & Charytan et al., ${ }^{28}$ \\
\hline eGFR $15-29 \mathrm{~mL} / \mathrm{min} / 1.73 \mathrm{~m}^{2}$ & Charytan et al., ${ }^{28}$ \\
\hline \multicolumn{2}{|c|}{ Metformin is associated with increased risk of worsening renal function } \\
\hline eGFR $15-30 \mathrm{~mL} / \mathrm{min} / 1.73 \mathrm{~m}^{2}$ & Hsu et al., ${ }^{27}$ \\
\hline $\mathrm{eGFR}<15 \mathrm{~mL} / \mathrm{min} / 1.73 \mathrm{~m}^{2}$ & Hsu et al., ${ }^{27}$ \\
\hline
\end{tabular}

Superscript numbers: Refer to REFERENCES

investigated 65 T2DM patients with CKD in whom metformin had been recently discontinued, and found a significant decrease in median lactate levels after the discontinuation of metformin but no significant difference in blood $\mathrm{pH}$, bicarbonate levels or base excess. Hung et al..$^{22}$ reported no significant difference in the incidence of metabolic acidosis in T2DM patients with CKD stage 5 receiving metformin compared with those not receiving metformin. In a study by Ekström et al., ${ }^{23}$ the authors reported no increased risk of acidosis in T2DM patients with CKD taking metformin compared with those taking other glucoselowering medications. Lazarus et al. ${ }^{25}$ reported no increased risk of acidosis with metformin use in CKD stage $3 \mathrm{a}$ or stage $3 \mathrm{~b}$. There was an increased risk of acidosis with metformin use in CKD stage 4 and above, but this was found to be not statistically significant. Bipi et al, ${ }^{29}$ reported no significant difference in arterial $\mathrm{pH}$, serum bicarbonate and serum lactate levels between 57 patients on metformin and 54 patients not on metformin. Richy et al. ${ }^{26}$ reported no significant difference in lactic acidosis incidence rate among people receiving metformin who had normal (no CKD or CKD stage 1), mildly reduced (CKD stage 2), moderately reduced (CKD stage 3 ) or severely reduced renal function (CKD stages 4 and 5).

In contrast, Eppenga et al. ${ }^{24}$ reported an increased risk of developing lactic acidosis or a high plasma lactate 
level in participants with CKD using metformin compared with those not using metformin. However, they did not distinguish between participants who developed lactic acidosis and participants who were found to have a high plasma lactate level without acidosis. It was not established in this cohort study that metformin use was significantly associated with the development of lactic acidosis.

\section{Impact on renal function}

The included studies presented conflicting findings of the impact of metformin use on renal function in T2DM patients with CKD. Two cohort studies 22,28 reported an association between metformin use and worsening renal function. Hsu et al. ${ }^{27}$ reported that continuation of metformin is a risk factor for worsening renal function in patients with CKD stages 4 and 5 . Similarly, in a retrospective cohort study spanning 10 years, Hung et al. ${ }^{22}$ reported that patients with CKD stage 5 using metformin were more likely to develop end-stage renal disease requiring dialysis than those who were not using metformin. In contrast, Charytan et al. ${ }^{28}$ reported that metformin use in patients with CKD stages $3 \mathrm{a}-4$ was associated with a lower risk of progression to end-stage renal disease or death from renal causes over a study period of 4 years.

\section{DISCUSSION}

The 14 included articles provide a moderate level of evidence to suggest that metformin may be associated with some benefits in T2DM patients with CKD. These benefits include improved all-cause mortality and reduced risk of cardiovascular events. There appears to be a low risk of lactic acidosis associated with metformin use in CKD. Metformin appears to confer these benefits in less severe stages of CKD (stage 4 and above), whereas the risks of increased mortality and progression of CKD are more prominent in CKD stage 5 .

One of the key concerns about the safety of metformin use in CKD is the risk of precipitating lactic acidosis through the excessive accumulation of lactate, as happened with a related drug (phenformin) which was taken off the market in $1978 .{ }^{11}$ The risk of lactic acidosis in metformin use may be overstated, and thus metformin use in CKD may be safer than previously thought. It has been highlighted that there are a large number of patients with CKD using metformin, yet lactic acidosis is rare. ${ }^{14}$ Lactic acidosis is generally associated with acute severe illness that causes excessive production and reduced ability to oxidise lactate (e.g. ischaemia or hypoxia) ${ }^{11}$; it may be that lactic acidosis is more related to these states of acute illness than to the use of metformin in CKD with no other acute metabolic derangements. Perhaps in weighing the risks and benefits of using metformin in a population with a reduced eGFR, the risk of lactic acidosis ought not to carry so much weight as it does in present guidelines.

A question remains without a clear answer: at what eGFR should we reduce the dose of metformin, and at what dose should we stop it altogether? Current guidelines advise to reduce the dose of metformin when eGFR is below $45 \mathrm{~mL} / \mathrm{min} / 1.73 \mathrm{~m}^{2}$ and stop metformin when eGFR is below $30 \mathrm{~mL} / \mathrm{min} / 1.73 \mathrm{~m}^{2}$. However, as there has been a report of improved mortality and cardiovascular outcomes even in a population with eGFRs as low as $20 \mathrm{~mL} / \mathrm{min} / 1.73 \mathrm{~m}^{2},{ }^{18}$ perhaps it is worth considering whether this eGFR threshold should be lowered. This lowering can be accompanied by frequent monitoring of eGFR to ensure that metformin is stopped should the eGFR deteriorate below this threshold. Currently, the guidelines of NICE and Kidney Disease: Improving Global Outcomes suggest monitoring renal function 3 to 4 times a year or more in stages 4 and 5 renal impairment. ${ }^{35,36}$ However, there appears to be a lack of evidence on how often to monitor for CKD progression specifically in patients with CKD stage 4 using metformin.

The use of metformin in severe renal impairment (CKD stage 5) appears to be detrimental, with an increased mortality ${ }^{22}$ and increased risk of CKD progression $^{22,27}$ associated with metformin use in CKD stage 5. The benefits of metformin use in CKD appear to be confined to CKD stage 4 and above. This position was echoed in the European Renal Best Practice guidelines in 2015, in which the authors considered the cost-benefit of metformin use in CKD stage 4 and beyond to be positive. However, they also acknowledged a lack of data on the safety of metformin use. Thus, like NICE, the American Association of Clinical Endocrinologists and the American College of Endocrinology, they advocated caution in using metformin in CKD stages 4 and 5. In this guideline, they recommended a dose reduction in metformin in CKD stage 4 and above, but reported that there was insufficient data regarding metformin use in such advanced stages of CKD. ${ }^{37}$

\section{Implication for clinical care}

The implication for clinical care is that the use of metformin at lower eGFRs should perhaps be considered, 
as metformin may have benefits for T2DM patients with CKD even with CKD stage 4. If the fears of lactic acidosis have been overestimated, the benefits could outweigh the risks.

\section{Strengths of this review}

This review provides an updated re-examination of the evidence for the benefits and risks of metformin use in T2DM patients with CKD, as guidelines have previously highlighted the lack of evidence for the safety of metformin in renal impairment. It includes studies conducted in the last 5 years since the publication of the most recent NICE guidelines on the matter, thus providing an update on the subject. In particular, it presents the latest findings on the effect of metformin use on all-cause mortality and cardiovascular events, as it includes very recent cohort studies and systematic reviews from the last 2 years.

\section{Limitations of this review}

Only published studies in English were included, and thus the possibility of publication bias cannot be excluded. Studies that failed to find significant results could have been published in local, non-English language journals, or may not have been published. There was also a dearth of randomised controlled trials investigating the benefits and risks of metformin in CKD.

\section{Directions for future research}

This review also highlights unanswered questions to which further research efforts can be devoted. Further research is needed to investigate whether metformin can still be used safely in advanced CKD (i.e. stage 5), and the eGFR threshold below which metformin can be stopped. Randomised controlled trials comparing metformin use with non-use in participants with advanced CKD may provide more information about whether the risk of adverse effects is increased with metformin use in very low eGFRs, or whether metformin use confers any benefits. To further discern the eGFR threshold for safe metformin use, cohort studies comparing the rate of adverse events in groups of participants with different stages of advanced CKD (e.g. adverse events in participants with CKD stage 4 on metformin versus those with CKD stage 5 on metformin) could be carried out.

\section{CONCLUSION}

This evidence-based review demonstrates that there is a moderate level of evidence to support the benefits of metformin use on reducing mortality and cardiovascular outcomes in T2DM patients with CKD stage 4 and above. There may not be a significant association between metformin use in renal impairment and lactic acidosis, as previously feared. Metformin use in CKD stage 5 may be associated with worse outcomes in mortality and CKD progression.

The results of this evidence-based review suggest that the previous recommendation to reduce the dosage of metformin in eGFRs of less than $45 \mathrm{~mL} /$ $\mathrm{min} / 1.73 \mathrm{~m}^{2}$ (corresponding to stage $3 \mathrm{a}$ ) may need to be reconsidered. There is a possibility that, in limiting the use of metformin in T2DM patients with stage 3 or $4 \mathrm{CKD}$, they are deprived of the benefits of metformin use.

\section{Acknowledgement}

The authors thank colleagues at the Family Medicine Service, KK Women's and Children's Hospital, for their helpful advice during the writing of this review.

\section{REFERENCES}

1. World Health Organization. Diabetes. Available at: https://www.who. int/health-topics/diabetes\#tab=tab_1. Accessed on 18 April 2020.

2. World Health Organization. Global Report on Diabetes, 21 April 2016. Available at: https://www.who.int/publications/i/ item/9789241565257. Accessed on 18 April 2020.

3. National Institute for Health and Care Excellence. Chronic kidney disease in adults: Assessment and management, 23 July 2014. Available at: https://www.nice.org.uk/guidance/cg182. Accessed on 24 April 2020.

4. Neuen BL, Chadban SJ, Demaio AR, et al. Chronic kidney disease and the global NCDs agenda. BMJ Glob Health 2017;2:e000380.

5. Song R. Mechanism of metformin: A tale of two sites. Diabetes Care 2016;39:187-9.

6. Corcoran C, Jacobs TF. Metformin. In: StatPearls. Treasure Island (FL): StatPearls Publishing; 2020.

7. Yoon H, Cho HY, Yoo HD, et al. Influences of organic cation transporter polymorphisms on the population pharmacokinetics of metformin in healthy subjects. AAPS J 2013;15:571-80.

8. National Institute for Health and Care Excellence. Type 2 diabetes in adults: Management, December 2015. Available at: https://www. nice.org.uk/guidance/ng28/evidence/full-guideline-pdf-78671532569. Accessed on 18 April 2020.

9. Goh SY, Ang SB, Bee YM, et al. Ministry of Health clinical practice guidelines: Diabetes mellitus. Singapore Med J 2014;55:334-47.

10. Garber AJ, Handelsman Y, Grunberger G, et al. Consensus statement by the American Association of Clinical Endocrinologists and American College of Endocrinology on the comprehensive type 2 diabetes management algorithm - 2020 executive summary. Endocr Pract 2020;26:107-39. 
11. MacCallum L, Senior PA. Safe use of metformin in adults with type 2 diabetes and chronic kidney disease: Lower dosages and sick-day education are essential. Can J Diabetes 2019;43:76-80.

12. van Dalem J, Brouwers MC, Stehouwer CD, et al. Risk of hypoglycaemia in users of sulphonylureas compared with metformin in relation to renal function and sulphonylurea metabolite group: Population based cohort study. BMJ 2016;354:i3625.

13. Russell-Jones D, Khan R. Insulin-associated weight gain in diabetes - causes, effects and coping strategies. Diabetes Obes Metab 2007;9:799-812.

14. Pilmore HL. Review: Metformin: Potential benefits and use in chronic kidney disease. Nephrology (Carlton) 2010;15:412-8.

15. Imam TH. Changes in metformin use in chronic kidney disease. Clin Kidney J 2017;10:301-4.

16. De Broe ME, Kajbaf F, Lalau JD. Renoprotective effects of metformin. Nephron 2018;138:261-74.

17. Crowley MJ, Diamantidis CJ, McDuffie JR, et al. Clinical outcomes of metformin use in populations with chronic kidney disease, congestive heart failure, or chronic liver disease: A systematic review. Ann Intern Med 2017;166:191-200.

18. Lu WR, Defilippi J, Braun A. Unleash metformin: Reconsideration of the contraindication in patients with renal impairment. Ann Pharmacother 2013;47:1488-97.

19. Hu Y, Lei M, Ke G, et al. Metformin use and risk of all-cause mortality and cardiovascular events in patients with chronic kidney disease: A systematic review and meta-analysis. Front Endocrinol (Lausanne) 2020;11:559446.

20. Whitlock RH, Hougen I, Komenda P, et al. A safety comparison of metformin vs sulfonylurea initiation in patients with type 2 diabetes and chronic kidney disease: A retrospective cohort study. Mayo Clin Proc 2020;95:90-100.

21. Marcum ZA, Forsberg CW, Moore KP, et al. Mortality associated with metformin versus sulfonylurea initiation: A cohort study of veterans with diabetes and chronic kidney disease. J Gen Intern Med 2018;33:155-65.

22. Hung SC, Chang YK, Liu JS, et al. Metformin use and mortality in patients with advanced chronic kidney disease: National, retrospective, observational, cohort study. Lancet Diabetes Endocrinol 2015;3:605-14.

23. Ekström N, Schiöler L, Svensson AM, et al. Effectiveness and safety of metformin in 51675 patients with type 2 diabetes and different levels of renal function: A cohort study from the Swedish National Diabetes Register. BMJ Open 2012;2:e001076.

24. Eppenga WL, Lalmohamed A, Geerts AF, et al. Risk of lactic acidosis or elevated lactate concentrations in metformin users with renal impairment: A population-based cohort study. Diabetes Care 2014;37:2218-24

25. Lazarus B, Wu A, Shin JI, et al. Association of metformin use with risk of lactic acidosis across the range of kidney function: A community-based cohort study. JAMA Intern Med 2018;178:903-10.

26. Richy FF, Sabidó-Espin M, Guedes S, et al. Incidence of lactic acidosis in patients with type 2 diabetes with and without renal impairment treated with metformin: A retrospective cohort study. Diabetes Care 2014;37:2291-5

27. Hsu WH, Hsiao PJ, Lin PC, et al. Effect of metformin on kidney function in patients with type 2 diabetes mellitus and moderate chronic kidney disease. Oncotarget 2017;9:5416-23.

28. Charytan DM, Solomon SD, Ivanovich P, et al. Metformin use and cardiovascular events in patients with type 2 diabetes and chronic kidney disease. Diabetes Obes Metab 2019;21:1199-208.

29. Bipi PK, George J, Gomathy S, et al. Lactate levels and risk of lactic acidosis with metformin in diabetic kidney disease patients. Saudi J Kidney Dis Transpl 2017;28:1356-61.

30. Sipahi S, Solak Y, Acikgoz SB, et al. Retrospective analysis of lactic acidosis-related parameters upon and after metformin discontinuation in patients with diabetes and chronic kidney disease. Int Urol Nephrol 2016;48:1305-12.

31. Inzucchi SE, Lipska KJ, Mayo H, et al. Metformin in patients with type 2 diabetes and kidney disease: A systematic review. JAMA 2014;312:2668-75.

32. Song JW, Chung KC. Observational studies: Cohort and case-control studies. Plast Reconstr Surg 2010;126:2234-42.

33. Camm AJ, Fox KAA. Strengths and weaknesses of 'real-world' studies involving non-vitamin $\mathrm{K}$ antagonist oral anticoagulants. Open Heart 2018;5:e000788.

34. Euser AM, Zoccali C, Jager KJ, et al. Cohort studies: Prospective versus retrospective. Nephron Clin Pract 2009;113:c214-7.

35. National Institute for Health and Care Excellence. Assessment and monitoring of chronic kidney disease, August 2014 Available at: https:// www.nice.org.uk/guidance/cg182. Accessed on 4 May 2020.

36. Kidney Disease: Improving Global Outcomes. KDIGO 2012 clinical practice guideline for the evaluation and management of chronic kidney disease. Kidney Int Suppl 2013;3:1-150.

37. Bilo $\mathrm{H}$, Coentrão L, Couchoud $\mathrm{C}$ et al. Clinical Practice Guideline on management of patients with diabetes and chronic kidney disease stage $3 \mathrm{~b}$ or higher (eGFR $<45 \mathrm{~mL} / \mathrm{min}$ ). Nephrol Dial Transplant 2015;30(Suppl 2):ii1-142.

38. Harbour R, Miller J. A new system for grading recommendations in evidence based guidelines. BMJ 2001;323:334-6. 